\title{
Análisis de los criterios de importancia y motivación para la elección de centros deportivos en función de variables sociodemográficas \\ Analysis of importance and motivation criteria for choosing sports centres according to socio- demographic variables
}

\author{
*Daniel Martínez-Cevallos, **Mario Alguacil, *Fernando García-Pascual
}

*Universidad de Valencia (España), *Universidad Católica de Valencia (España)

\begin{abstract}
Resumen. El presente artículo pretende analizar qué aspectos consideran importantes los usuarios de centros deportivos y qué elementos les motivan a inscribirse en ellos, atendiendo a características sociodemográficas como el género, el nivel de estudios, la ocupación o el nivel socioeconómico. Para ello, se han analizado las opiniones de usuarios actuales y potenciales de centros deportivos, preguntándoles acerca de los aspectos comentados anteriormente, ofreciendo una escala de respuesta tipo Likert de 5 puntos. Los resultados indican que la localización del centro, así como que el centro tenga entrenadores personales, actividades dirigidas y que sea multidisciplinar, son elementos importantes para ellos. Por otro lado, también se observa que la higiene, la amabilidad de los entrenadores, el equipamiento o el nivel tecnológico del centro, son los elementos que les motivan a la hora de elegir un centro deportivo. Por tanto, conociendo los criterios de importancia y motivación, los gestores disponen de información útil para poder orientar de manera más efectiva sus estrategias de marketing, con la intención de ajustarse en mayor medida a las demandas e intereses de los usuarios, mejorando la satisfacción de los usuarios actuales y aumentando las probabilidades de elección por parte de los potenciales.

Palabras clave: importancia, motivación, servicios deportivos, centros fitness, gestión deportiva.
\end{abstract}

\begin{abstract}
This article aims to analyse which aspects are considered important by users of sports centres and which elements motivate them to join such centres, considering socio-demographic characteristics such as gender, educational level, occupation or socio-economic level. For this purpose, the opinions of current and potential users of sports centres have been analysed, asking them about the aspects mentioned above, offering a 5-point Likert type response scale. The results indicate that centre location, as well as the fact that the centre has personal trainers, guided activities and that it is multidisciplinary, are important elements for them. On the other hand, it is also observed that hygiene, the kindness of trainers, the equipment or the technological level of the centre, are the elements that motivate them when choosing a sports centre. Therefore, knowing the importance and motivation criteria, managers have useful information to be able to orient their marketing strategies more effectively, with the intention of adjusting to the demands and interests of users to a greater extent, improving the satisfaction of current users and increasing the probabilities of choice by potential users.
\end{abstract}

Keywords: importance, motivation, sports services, fitness centres, sports management.

\section{Introducción}

El incremento que se ha producido en relación a la práctica de actividad física, ha supuesto lógicamente un aumento del número de personas inscritas en centros deportivos y organizaciones deportivas. La oferta de servicios deportivos ha ido creciendo en consonancia, para dar respuesta a esa creciente demanda, surgiendo la posibilidad de crear distintos tipos de negocio con fines lucrativos, pero también sin ánimo de lucro, tratando de que las entidades tuvieran fines sociales (Nuviala et al., 2014). Esa variedad y diversidad de oferta que se ha ido creando, se ha producido tanto en los servicios públicos como privados, con el fin de satisfacer la demanda deportiva, una demanda que ha ido alcanzando dimensiones culturales, sociales y de mercado que hasta ahora no se conocían y que cada vez plantea escenarios más complejos (Ros-Castelló, Calabuig \& Tafalla, 2019).

En este sentido, dada la amplia oferta y tipologías de usuarios, el conocimiento delimitado de cada tipología o grupos de usuarios contribuirá a conocer mejor sus intereses y, por tanto, aporta información para poder tratar de incrementar la satisfacción del usuario, su fidelización, así como el tiempo de permanencia en la organización (Brady et al., 2005). Siguiendo esta línea, y por los motivos anteriormente comentados de diversificación de la oferta, en los últimos años

Fecha recepción: 14-05-20. Fecha de aceptación: 15-07-20

Mario Alguacil

mario.alguacil@ucv.es ha surgido una mayor necesidad de analizar los perfiles de los usuarios de instalaciones deportivas, como herramienta estratégica para conocer en profundidad dichos perfiles y poder adecuar la oferta del servicio a la demanda real, siendo más competentes respecto a la competencia del mercado (Nuviala et al., 2014). En todos los contextos, y en concreto en el deportivo que es el que nos ocupa, este análisis de los perfiles de usuario, así como la segmentación de clientes, se llevan a cabo con el fin de facilitar a los centros información que les permita aportar un valor añadido y poder diferenciarse de los demás. Con dicha información, los centros deportivos pueden establecer políticas de precios y estrategias de marketing más adecuadas a su target (Molina, Mundina \& Gómez, 2019) además de conocer en mayor medida sus características específicas (García-Fernández, Gálvez, Bernal \& Vélez, 2016).

En este tipo de estudios, encontramos diferentes enfoques centrados, por ejemplo, en el análisis del mercado, con la intención de comprobar el efecto del tipo de cuota como variable de fidelización de los usuarios (Triadó y Aparicio, 2004). Por otro lado, encontramos aportaciones que atienden al análisis de las motivaciones de los usuarios (LunaArocas, 1998; Luna-Arocas, Mundina y Quintanilla, 1997; Martínez y Balbastre, 2004; Mundina y Calabuig, 1999) mientras que, finalmente, también observamos contribuciones que atienden al análisis del perfil de usuario basándose, como en el caso de la presente investigación, en el análisis en función de los aspectos sociodemográficos (Desbordes, Ohl \& Tribou 2001; García-Fernández, Fernández-Gavira \& Bernal, 
2014; Luna-Arocas \& Li-ping, 2005; Rial, Lamas \& Varela, 2009) entre los que encontrados el género, la edad, ocupación, nivel de estudios o nivel socioeconómico.

\section{Motivos e Importancia para elegir un Centro Deporti-} vo

En el ámbito deportivo, a pesar de que el mundo del fitness ha tenido un crecimiento exponencial a nivel global, en la literatura se encuentra información muy limitada sobre el estudio de los motivos que hacen a los usuarios elegir un centro deportivo. En este sentido, encontramos estudios como el de Frederick y Ryan (1993) en el que analizan relaciones entre ejercicio físico y la motivación y la conducta autoconfirmada, teniendo en cuenta aspectos como la duración y frecuencia con la que los usuarios realizaban actividad física. Por otro lado, aportaciones como la de Ryan, Frederick, Lepes, Rubio y Sheldon (1997) realizaron estudios en centros fitness, con el objetivo de analizar de qué manera los motivos de adherencia predecían la práctica de actividad física. Por su parte, Thogersen, Lane, Biscomb, Jarrett y Lane (2007) analizaron las relaciones entre los motivos para la práctica de ejercicio físico y la conducta en mujeres, atendiendo también a aspectos relacionados con su apariencia física o la competición (Sicilia et al., 2014). En este sentido, los estudios sobre motivaciones van unidos a elementos como el bienestar o la salud, sin embargo, no son comunes los estudios que indiquen información sobre las motivaciones hacia las instalaciones, equipamientos, horarios o precios, aspectos que también pueden ser interesantes para que los usuarios decidan asistir a un centro deportivo $u$ otro. Cuando el sector de los centros deportivos no era un sector potente y dinámico como lo es actualmente, era el usuario quien debía adaptarse a las condiciones que ofrecía el servicio deportivo. Actualmente, ese orden establecido se ha invertido, y es el centro deportivo el que adapta sus servicios para satisfacer a sus perfiles de interés. Por ello, de un tiempo a esta parte, encontramos cada vez más trabajos que analizan los perfiles de los usuarios que acuden a las instalaciones deportivas (Campos, Martins, Simões \& Franco, 2017; LéonQuismondo, García-Unanue \& Burillo, 2020) lo que permite obtener datos más precisos que ayuden a mejorar cualitativamente el servicio deportivo prestado. Por tanto, el análisis de los intereses de los distintos perfiles de usuarios, permite conocer la importancia que otorgan a los diferentes aspectos del servicio, por lo que contribuye a que los gestores puedan entender sus elecciones como consumidor y puedan reforzar esos aspectos para mejorar su fidelidad.

Por ello, el estudio de la importancia y valoración, que originariamente surgió en el campo del marketing pero que ya se ha extendido a numerosos contextos, es una parte imprescindible en el análisis de los usuarios de centros deportivos. Su importancia reside en que permite a los gestores identificar errores y comprobar de qué manera los esfuerzos que se están realizando están orientados a los aspectos importantes de la manera adecuada para, de esa forma, poder llevar a cabo las adaptaciones que pudieran ser necesarias. Conocer qué atributos son importantes y de qué manera los valoran los usuarios, será determinante para poder tratar de mejorarlos, con el objetivo de tener clientes satisfechos y fieles (Zamorano-Solís \& García-Fernández, 2018). Por los motivos expuestos, el objetivo del presente estudio es analizar los diferentes criterios que los usuarios de centros deportivos consideran importantes, así como los motivos que los llevan a decidir inscribirse en un centro deportivo, analizando dichos aspectos en función de las características sociodemográficas. Ese tipo de análisis permitirá conocer las opiniones de los distintos perfiles de usuario, al mismo tiempo que supone una contribución a la literatura científica, ya que en el ámbito deportivo el análisis del perfil del usuario es todavía una temática en fase de desarrollo.

\section{Método}

\section{Muestra}

La población objeto de este estudio la constituyeron los habitantes, mujeres y hombres residentes en el norte de la capital de Ecuador Quito-Cumbayá y los cantones de alrededor. El criterio de selección de la muestra consistió en que los encuestados fueran usuarios de centros deportivos o lo hubieran sido, además de que fuesen mayores de edad. En este sentido la edad de los participantes se movió en un rango entre 18 y 65 años. Con el objetivo de asegurar un mayor tamaño muestral de la población objeto de estudio, se ha obtenido la muestra mediante un muestreo no probabilístico intencional o de conveniencia, consiguiendo un total de 116 encuestas válidas. Del total de la muestra, el $49 \%$ son mujeres y el $51 \%$ hombres. En cuanto a la edad, la media ha sido de 36 años, siendo la moda de 24 años, con un rango de edad de los encuestados de 19 a 61 años. Por otro lado, respecto al nivel de estudios, la gran mayoría tiene estudios universitarios (88.3\%) seguido por el $11.7 \%$ que posee estudios secundarios. En cuanto a la ocupación, la mayor parte de los participantes cuentan con un empleo, lo que supone el $82 \%$ de la muestra, seguido por los estudiantes $(11.7 \%)$ los jubilados $(3.6 \%) \mathrm{y}$, por último, los desempleados $(2.7 \%)$. Finalmente, respecto al nivel socioeconómico, la mayoría se considera de nivel medio $(81.1 \%)$, mientras que un $16.2 \%$ considera que tiene un nivel bajo y solamente el $2.7 \%$ opina tener un nivel alto.

\section{Instrumento}

El instrumento para la recogida de la información fue un cuestionario estructurado en 4 bloques, que conformaban un total de 20 enunciados con respuesta tipo Likert, donde 1 significaba estar completamente en desacuerdo y 5 completamente de acuerdo con esas afirmaciones. Para la creación de dicho cuestionario, se ha tomado como referencia la escala QSport de Rial, Rial, Varela y Real (2008) indicando los criterios de motivación e importancia. A dichos enunciados se les sumaban unas últimas cuestiones orientadas a recoger la información sociodemográfica de los usuarios. En la primera parte del cuestionario, se pregunta acerca de los hábitos de uso de instalaciones deportivas, así como sobre las preferencias respecto al tipo de instalación deportiva. En segundo lugar, se pregunta acerca de cómo han obtenido información de los centros deportivos a los que se han inscrito, mientras que en el bloque 3 se pregunta acerca de su comportamiento, obteniendo información de aspectos como su antigüedad como usuario o los motivos que los han llevado a abandonar un centro deportivo. Finalmente, el bloque 4, 
en el que se fundamenta este estudio, recoge la información referida a los criterios de importancia que según los encuestados debe tener un centro deportivo, así como los aspectos relacionados con sus motivaciones para decidir inscribirse o no en un centro deportivo. La última parte, como se comentaba al inicio, recogía la información relacionada con las variables sociodemográficas.

\section{Procedimiento y análisis estadístico}

Este cuestionario fue digitalizado y subido a la plataforma electrónica LimeSurvey para, posteriormente, poder ser enviada por correo electrónico y redes sociales a los participantes del estudio. En cuanto al tiempo para rellenar el cuestionario, el proceso tenía una duración aproximada de entre 8 y 10 minutos. Para el análisis de los datos se utilizó el software SPSS versión 25, donde se realizó, en primer lugar, un análisis descriptivo para conocer la media obtenida de las distintas variables en función de las características sociodemográficas. Posteriormente, se llevaron a cabo comparaciones de medias mediante pruebas T y ANOVA, para comprobar si las medias obtenidas entre los distintos grupos de interés mostraban diferencias significativas. El presente artículo analiza qué aspectos son importantes y motivan a los usuarios de centros deportivos, según características sociodemográficas como el género, el nivel de estudios, la ocupación o el nivel socioeconómico, con la intención de conocer posibles diferencias en función del grupo de pertenencia. Para solventar este aspecto, en las pruebas Post Hoc se ha utilizado la prueba de Hochberg, que tiene en cuenta la posible pérdida de homogeneidad en el tamaño de los grupos generados.

\section{Resultados}

\section{Criterios de importancia}

\section{Criterios de importancia según el género}

En cuanto a los resultados de los criterios de importancia según el género (ver tabla 1), existen variables tales como la cercanía del centro al domicilio $(4.48 \pm .66)$ y que sea multidisciplinar ( $4.38 \pm .98)$, que en el caso de los hombres son las que ofrecen los valores medios más altos, mientras que los valores más bajos aparecen en que el centro sea pequeño $(2.16 \pm 1.39)$ y que esté cerca de un centro comercial $(2.41 \pm 1.37)$. En el caso de las mujeres, los aspectos con una media más elevada son que esté cerca del domicilio (4.60 $\pm .71)$ y que tenga servicio de entrenador personal (4.58 \pm .83$)$. En cambio, los elementos a los que conceden menos importancia son que sea público $(2.58 \pm 1.41)$ y que esté en un centro comercial $(2.46 \pm 1.42)$. En cuanto a la comparación por

\begin{tabular}{|c|c|c|c|c|c|c|c|c|c|}
\hline Variable & Género & $\mathrm{M}$ & DT & $\mathrm{P}$ & Variable & Género & $\mathrm{M}$ & DT & $P$ \\
\hline \multirow[b]{2}{*}{ Público } & Femenino & 2.58 & 1.41 & \multirow{2}{*}{\multicolumn{2}{|c|}{$\begin{array}{c}\text { Entrenador } \\
\text { personal }\end{array}$}} & Femenino & 4.58 & .83 & \multirow{2}{*}{$*$} \\
\hline & Masculino & 2.63 & 1.48 & & & Masculino & 4.13 & 1.11 & \\
\hline \multirow{2}{*}{ Privado } & Femenino & 3.80 & 1.24 & \multirow{2}{*}{\multicolumn{2}{|c|}{$\begin{array}{c}\text { Actividades } \\
\text { dirigidas }\end{array}$}} & Femenino & 4.51 & .81 & \\
\hline & Masculino & 3.82 & 1.35 & & & Masculino & 4.00 & 1.14 & \\
\hline \multirow[b]{2}{*}{ Grande } & Femenino & 4.11 & .99 & \multirow{2}{*}{\multicolumn{2}{|c|}{$\begin{array}{c}\text { Cerca } \\
\text { domicilio }\end{array}$}} & Femenino & 4.60 & .71 & \\
\hline & Masculino & 4.23 & .89 & & & Masculino & 4.48 & .66 & \\
\hline \multirow{2}{*}{ Pequeño } & Femenino & 2.18 & 1.32 & \multirow{2}{*}{\multicolumn{2}{|c|}{ Cerca trabajo }} & Femenino & 3.53 & \multicolumn{2}{|l|}{1.49} \\
\hline & Masculino & 2.16 & 1.39 & & & Masculino & 3.43 & 1.37 & \\
\hline \multirow{2}{*}{ Multidisciplinar } & Femenino & 4.53 & .74 & \multirow{2}{*}{\multicolumn{2}{|c|}{$\begin{array}{l}\text { Centro } \\
\text { comercial }\end{array}$}} & Femenino & 2.46 & \multicolumn{2}{|l|}{1.42} \\
\hline & Masculino & 4.38 & .98 & & & Masculino & 2.41 & 1.37 & \\
\hline \multirow{2}{*}{ Unidisciplinar } & Femenino & 2.64 & 1.48 & \multirow{2}{*}{\multicolumn{2}{|c|}{$\begin{array}{c}\text { Alejado } \\
\text { centro }\end{array}$}} & Femenino & 2.69 & \multicolumn{2}{|l|}{1.37} \\
\hline & Masculino & 2.46 & 1.50 & & & Masculino & 3.11 & 1.47 & \\
\hline \multirow{2}{*}{ Guía } & Femenino & 4.42 & 1.05 & & & & & & \\
\hline & Masculino & 4.14 & 1.15 & & & & & & \\
\hline
\end{tabular}

género, encontramos diferencias significativas en la importancia que le conceden a los distintos aspectos en las variables de que el centro tenga entrenador personal $(\mathrm{p}<.05)$ y que tenga actividades dirigidas $(\mathrm{p}<.01)$, en ambos casos con una media más elevada en las mujeres.

\section{Criterios de importancia según nivel de estudios}

Atendiendo a los criterios de importancia para elegir un centro deportivo, en este caso según el nivel de estudios, vemos en la tabla 2 que, para aquellos con estudios secundarios, el aspecto más importante es que el centro sea multidisciplinar (4.54 \pm .78 ) y el menos valorado que el centro deportivo sea pequeño $(3.00 \pm 1.47)$. Por otro lado, en el caso de los estudiantes universitarios, encontramos como aspecto más valorado, que el centro esté cerca del domicilio (4.56 $\pm .67)$ y como elemento menos importante para ellos, que el centro sea pequeño $(2.06 \pm 1.30)$. En cuanto a la comparación en función del nivel de estudios, vemos que existen diferencias en cuanto a que el centro sea público $(p<.01)$ y que sea pequeño $(\mathrm{p}<.05)$ donde los que tienen estudios secundarios, les conceden más importancia.

\begin{tabular}{|c|c|c|c|c|c|c|c|c|}
\hline Variable & Estudios & $\mathrm{M}$ & DT & $\mathrm{P}$ & Variable & Estudios & M & DT \\
\hline \multirow{2}{*}{ Público } & Secundarios & 3.62 & 1.61 & & Entrenador & Secundarios & 4.31 & 1.03 \\
\hline & Universitarios & 2.47 & 1.37 & Fit & personal & Universitarios & 4.36 & 1.01 \\
\hline \multirow{2}{*}{ Privado } & Secundarios & 3.54 & 1.51 & & Actividades & Secundarios & 4.31 & 1.03 \\
\hline & Universitarios & 3.85 & 1.26 & & dirigidas & Universitarios & 4.25 & 1.03 \\
\hline \multirow{2}{*}{ Grande } & Secundarios & 4.15 & 1.07 & & Cerca & Secundarios & 4.39 & .77 \\
\hline & Universitarios & 4.17 & .93 & & domicilio & Universitarios & 4.56 & .67 \\
\hline \multirow{2}{*}{ Pequeño } & Secundarios & 3.00 & 1.47 & & Cerca & Secundarios & 4.00 & 1.08 \\
\hline & Universitarios & 2.06 & 1.30 & * & trabajo & Universitarios & 3.41 & 1.46 \\
\hline \multirow{2}{*}{ Multidisciplina } & Secundarios & 4.54 & .78 & & Centro & Secundarios & 3.23 & 1.30 \\
\hline & Iniversitarios & 4.44 & .89 & & comercial & Universitarios & 2.33 & 1.38 \\
\hline \multirow{2}{*}{ Unidisciplinar } & Secundarios & 3.15 & 1.41 & & Alejado & Secundarios & 3.31 & 1.55 \\
\hline & Universitarios & 2.47 & 1.49 & & centro & Universitarios & 2.85 & 1.42 \\
\hline \multirow{2}{*}{ Guía } & Secundarios & 4.23 & .93 & & & & & \\
\hline & Universitarios & 4.29 & 1.13 & & & & & \\
\hline
\end{tabular}

Criterios de importancia según ocupación

El tercer aspecto analizado ha sido la ocupación (ver tabla 3). Aquí, encontramos que las personas empleadas otorgan la mayor importancia a que el centro esté cerca del domicilio (4.52 \pm .70$)$, mientras que el peor valorado es que el centro deportivo sea pequeño $(2.15 \pm 1.33)$. En el caso de los desempleados, los aspectos con media más alta y más baja son que el centro sea grande $(5.00 \pm .00)$ y multidisciplinar $(5.00 \pm .00)$, y que el centro sea público $(2.00 \pm 1.73)$ y peque-

\begin{tabular}{|c|c|c|c|c|c|c|c|c|}
\hline Variable & Situación & $\begin{array}{c}\text { Medi } \\
\text { a }\end{array}$ & DT & $\begin{array}{c}\mathrm{P} \\
\text { 1vs4 }\end{array}$ & Variable & Situación & $\mathrm{M}$ & DT \\
\hline \multirow{4}{*}{ Público } & Empleado & 2.53 & 1.45 & \multirow{4}{*}{\multicolumn{2}{|c|}{$\begin{array}{l}\text { Entrenador } \\
\text { personal }\end{array}$}} & Empleado & 4.32 & 1.04 \\
\hline & Desempleado & o 2.00 & 1.73 & & & Desempleado & 4.33 & .58 \\
\hline & Estudiante & 2.77 & 1.30 & & & Estudiante & 4.46 & .97 \\
\hline & Jubilado & 4.25 & .50 & & & Jubilado & 4.75 & .50 \\
\hline \multirow{4}{*}{ Privado } & Empleado & 3.76 & 1.30 & \multirow{4}{*}{\multicolumn{2}{|c|}{$\begin{array}{l}\text { Actividades } \\
\text { dirigidas }\end{array}$}} & Empleado & 4.30 & .97 \\
\hline & Desempleado & o 5.00 & .00 & & & Desempleado & 4.33 & .58 \\
\hline & Estudiante & 3.69 & 1.32 & & & Estudiante & 3.77 & 1.42 \\
\hline & Jubilado & 4.50 & 1.00 & & & Jubilado & 4.75 & .50 \\
\hline \multirow{4}{*}{ Grande } & Empleado & 4.10 & .98 & \multirow{4}{*}{\multicolumn{2}{|c|}{$\begin{array}{c}\text { Cerca } \\
\text { domicilio }\end{array}$}} & Empleado & 4.52 & .70 \\
\hline & Desempleado & o 5.00 & .00 & & & Desempleado & 4.67 & .58 \\
\hline & Estudiante & 4.54 & .66 & & & Estudiante & 4.62 & .65 \\
\hline & Jubilado & 4.00 & .82 & & & Jubilado & 4.75 & .50 \\
\hline \multirow{4}{*}{ Pequeño } & Empleado & 2.15 & 1.33 & \multirow{4}{*}{ * } & \multirow{4}{*}{ Cerca trabajo } & Empleado & 3.45 & 1.45 \\
\hline & Desempleado & o 2.00 & 1.73 & & & Desempleado & 4.33 & 1.15 \\
\hline & Estudiante & 1.69 & .95 & & & Estudiante & 3.77 & 1.30 \\
\hline & Jubilado & 4.25 & .96 & & & Jubilado & 2.50 & 1.29 \\
\hline \multirow{4}{*}{ Multidisciplinar } & Empleado & 4.45 & .89 & \multirow{4}{*}{\multicolumn{2}{|c|}{$\begin{array}{l}\text { Centro } \\
\text { comercial }\end{array}$}} & Empleado & 2.36 & 1.36 \\
\hline & Desempleado & o 5.00 & .00 & & & Desempleado & 3.67 & 1.53 \\
\hline & Estudiante & 4.31 & .85 & & & Estudiante & 2.62 & 1.45 \\
\hline & Jubilado & 4.50 & 1.00 & & & Jubilado & 2.50 & 1.91 \\
\hline \multirow{4}{*}{ Unidisciplinar } & Empleado & 2.47 & 1.47 & \multirow{4}{*}{ * } & \multirow{4}{*}{$\begin{array}{l}\text { Alejado } \\
\text { centro }\end{array}$} & Empleado & 2.90 & 1.45 \\
\hline & Desempleado & o 3.33 & 2.08 & & & Desempleado & 3.33 & 2.08 \\
\hline & Estudiante & 2.31 & 1.32 & & & Estudiante & 2.69 & 1.11 \\
\hline & Jubilado & 4.50 & .58 & & & Jubilado & 3.25 & 2.06 \\
\hline \multirow{4}{*}{ Guía } & Empleado & 4.28 & 1.11 & & & & & \\
\hline & Desempleado & o 4.67 & .58 & & & & & \\
\hline & Estudiante & 4.00 & 1.29 & & & & & \\
\hline & Jubilado & 5.00 & .00 & & & & & \\
\hline
\end{tabular}


ño $(2.00 \pm 1.73)$, respectivamente. Por otro lado, entre los estudiantes, el aspecto más importante que debe poseer un centro deportivo es que esté cerca del domicilio (4.62 \pm .65$)$, y el menos deseado que sea pequeño $(1.69 \pm .95)$. Finalmente, para los jubilados el aspecto más importante es que tenga servicio de entrenador personal (4.50 \pm .50$)$ actividades dirigidas $(4.50 \pm .50)$ y que esté cerca del domicilio $(4.75 \pm .50), \mathrm{y}$ el menos importante es que el centro esté cerca del trabajo $(2.50 \pm 1.29)$ lo que parece lógico por su situación, y que el centro esté en un centro comercial $(2.50 \pm 1.91)$. Respecto a las posibles diferencias entre las distintas situaciones laborales, encontramos diferencias significativas en la característica pequeño $(\mathrm{p}<.05)$ y unidisciplinar $(\mathrm{p}<.05)$.

Criterios de importancia según nivel socioeconómico

Finalmente, el último aspecto que se ha tenido en cuenta para el análisis de las opiniones de los encuestados ha sido su nivel socioeconómico (ver tabla 4). En este sentido, las personas con un nivel socioeconómico medio-bajo, muestran la media más alta en el aspecto de que el centro sea multidisciplinar $(4.83 \pm .38)$ y consideran que el hecho de que esté en un centro comercial $(2.67 \pm 1.37)$ es lo menos relevante. En cuanto a los encuestados de nivel socioeconómico medio, el aspecto al que más importancia conceden es que el centro esté cerca del domicilio $(4.52 \pm .69)$ mientras que el menos importante es que esté en un centro comercial (2.34 \pm 1.36 ). Finalmente, en cuanto a los de nivel socioeconómico alto, la media más elevada aparece en el aspecto de que el centro sea grande $(5.00 \pm .00)$ y esté cerca del domicilio (5.00 $\pm .00)$ mientras que la media más baja la encontramos en que esté alejado del centro $(2.33 \pm 2.31)$. En cuanto a la comparativa de grupos, no se encuentran diferencias estadísticamente significativas entre ninguno de ellos.

\begin{tabular}{|c|c|c|c|c|c|c|c|}
\hline Variable & Situación & M & DT & Variable & Situación & $\mathrm{M}$ & DT \\
\hline \multirow{3}{*}{ Público } & Medio-Bajo & 3.17 & 1.58 & \multirow{3}{*}{$\begin{array}{c}\text { Entrenador } \\
\text { personal }\end{array}$} & Medio-Bajo & 4.22 & 1.00 \\
\hline & Medio & 2.48 & 1.38 & & Medio & 4.39 & .99 \\
\hline & Alto & 3.00 & 2.00 & & Alto & 4.00 & 1.73 \\
\hline \multirow{3}{*}{ Privado } & Medio-Bajo & 3.61 & 1.42 & \multirow{3}{*}{$\begin{array}{l}\text { Actividades } \\
\text { dirigidas }\end{array}$} & Medio-Bajo & 4.33 & .91 \\
\hline & Medio & 3.81 & 1.27 & & Medio & 4.24 & 1.03 \\
\hline & Alto & 5.00 & .00 & & Alto & 4.00 & 1.73 \\
\hline \multirow{3}{*}{ Grande } & Medio-Bajo & 4.33 & .91 & \multirow{3}{*}{$\begin{array}{c}\text { Cerca } \\
\text { domicilio }\end{array}$} & Medio-Bajo & 4.56 & .70 \\
\hline & Medio & 4.11 & .95 & & Medio & 4.52 & .69 \\
\hline & Alto & 5.00 & .00 & & Alto & 5.00 & .00 \\
\hline \multirow{3}{*}{ Pequeño } & Medio-Bajo & 1.83 & 1.10 & \multirow{3}{*}{ Cerca trabajo } & Medio-Bajo & 3.56 & 1.29 \\
\hline & Medio & 2.23 & 1.37 & & Medio & 3.48 & 1.45 \\
\hline & Alto & 2.33 & 2.31 & & Alto & 3.00 & 2.00 \\
\hline \multirow{3}{*}{ Multidisciplinar } & Medio-Bajo & 4.83 & .38 & \multirow{3}{*}{$\begin{array}{l}\text { Centro } \\
\text { comercial }\end{array}$} & Medio-Bajo & 2.67 & 1.37 \\
\hline & Medio & 4.39 & .92 & & Medio & 2.34 & 1.36 \\
\hline & Alto & 4.00 & 1.00 & & Alto & 3.67 & 2.31 \\
\hline \multirow{3}{*}{ Unidisciplinar } & Medio-Bajo & 2.72 & 1.45 & \multirow{3}{*}{$\begin{array}{l}\text { Alejado } \\
\text { centro }\end{array}$} & Medio-Bajo & 3.28 & 1.45 \\
\hline & Medio & 2.49 & 1.49 & & Medio & 2.84 & 1.41 \\
\hline & Alto & 3.33 & 2.08 & & Alto & 2.33 & 2.31 \\
\hline \multirow{3}{*}{ Guía } & Medio-Bajo & 4.22 & 1.22 & & & & \\
\hline & Medio & 4.29 & 1.09 & & & & \\
\hline & Alto & 4.33 & 1.15 & & & & \\
\hline
\end{tabular}

\section{Criterios de motivación}

\section{Criterios de motivación en función del género}

Siguiendo con el análisis realizado anteriormente, pero en este caso con los criterios de motivación a la hora de elegir centro deportivo, en primer lugar, respecto al género (ver tabla 5), vemos que en el caso de las mujeres el aspecto que más les motiva para elegir un centro deportivo es que las instalaciones estén limpias $(4.86 \pm .45)$ mientras que el que menos les motiva es que el centro tenga cafetería social (3.33 \pm 1.49 ). Por otro lado, en el caso de los hombres, se repiten los mismos elementos, siendo la limpieza el principal motivo para unirse a un centro $(4.79 \pm .65)$ mientras que el elemento menos motivante es que tenga cafetería $(3.41 \pm 1.30)$. Respecto a la comparativa en función del género, no existen diferencias significativas entre hombres y mujeres en ninguna de las variables.

\begin{tabular}{|c|c|c|c|c|c|c|c|}
\hline Variables & Género & Media DT & Variables & Género & $\mathrm{N}$ & Media & DT \\
\hline \multirow{2}{*}{ Equipamiento } & Femenino & $\begin{array}{lll}4.40 \quad .87\end{array}$ & \multirow{2}{*}{ Campeonatos } & Femenino & 55 & 3.62 & 1.25 \\
\hline & Masculino & o 4.50 & & Masculino & 56 & 3.79 & 1.32 \\
\hline \multirow{2}{*}{ Variedad maquinaria } & Femenino & 4.55 & \multirow{2}{*}{$\begin{array}{c}\text { Alimentación } \\
\text { saludable }\end{array}$} & Femenino & 55 & 4.20 & 1.06 \\
\hline & Masculino & o 4.36 & & Masculino & 56 & 4.02 & 1.12 \\
\hline \multirow{2}{*}{ Última tecnología } & Femenino & $4.38 \quad .83$ & \multirow{2}{*}{ Cafetería social } & Femenino & 55 & 3.33 & 1.49 \\
\hline & Masculino & $\begin{array}{lll}0 & 4.18 \quad 1.03 \\
\end{array}$ & & Masculino & 56 & 3.41 & 1.30 \\
\hline \multirow{2}{*}{ Entrenadores titulado } & Femenino & $4.26 \quad .95$ & \multirow{2}{*}{$\begin{array}{l}\text { Instalaciones } \\
\text { limpias }\end{array}$} & Femenino & 55 & 4.86 & .45 \\
\hline & Masculino & $\begin{array}{lll} & 3.95 & 1.20 \\
\end{array}$ & & Masculino & 56 & 4.79 & .65 \\
\hline \multirow{2}{*}{ Entrenadores amables } & Femenino & $\begin{array}{lll}5.69 \quad .60 \\
\end{array}$ & \multirow{2}{*}{$\begin{array}{c}\text { Instalaciones } \\
\text { disponibles }\end{array}$} & Femenino & 55 & 4.56 & .63 \\
\hline & ${ }^{\mathrm{S}}$ Masculino & o 4.71 & & Masculino & 56 & 4.38 & .84 \\
\hline \multirow{2}{*}{ Zona social } & Femenino & $3.75 \quad 1.25$ & & & & & \\
\hline & Masculino & $\begin{array}{lll}\text { o } 3.86 & 1.24 \\
\end{array}$ & & & & & \\
\hline
\end{tabular}
dios

Criterios de motivación en función del nivel de estu-

En segundo lugar, con relación al nivel de estudios, como se puede apreciar en la tabla 6, encontramos que, en las personas con estudios secundarios, los aspectos que obtienen una media más alta son que el centro tenga entrenadores amables (4.85 \pm .38$)$ e instalaciones limpias (4.85 \pm .38 ), mientras que la media más baja aparece en que el centro tenga cafetería social $(4 \pm 1.00)$. Por otro lado, para el caso de los que poseen estudios universitarios, el aspecto más motivante es que las instalaciones estén limpias (4.82 \pm .58$)$ mientras que el menos motivante resulta ser, de nuevo, que el centro cuente con una cafetería social $(3.29 \pm 1.42)$. En la comparativa en función de los estudios que poseen los encuestados, encontramos diferencias significativas en el aspecto de que el centro de la opción de comprar comida saludable $(\mathrm{p}<.05)$.

\begin{tabular}{|c|c|c|c|c|c|c|c|}
\hline Variable & Estudios & Media & DT & Variable & Estudios & $\mathrm{N}$ & media DT P \\
\hline \multirow{2}{*}{ Equipamiento } & Secundarios & 4.46 & .66 & \multirow{2}{*}{ Campeonatos } & Secundarios & 13 & $\begin{array}{ll}4.23 \quad .93 \\
\end{array}$ \\
\hline & Universitarios & 4.45 & .84 & & Universitarios & 98 & 3.631 .31 \\
\hline \multirow{2}{*}{$\begin{array}{c}\text { Variedad } \\
\text { maquinaria }\end{array}$} & Secundarios & 4.62 & .65 & \multirow{2}{*}{$\begin{array}{l}\text { Alimentación } \\
\text { saludable }\end{array}$} & Secundarios & 13 & $\begin{array}{ll}4.69 & .85\end{array}$ \\
\hline & Universitarios & 4.43 & .92 & & Universitarios & 98 & $\begin{array}{lll}4.03 & 1.10 \\
\end{array}$ \\
\hline \multirow{2}{*}{$\begin{array}{c}\text { Última } \\
\text { tecnología }\end{array}$} & Secundarios & 4.39 & .51 & \multirow{2}{*}{$\begin{array}{c}\text { Cafetería } \\
\text { social }\end{array}$} & Secundarios & 13 & $\begin{array}{lll}4.00 & 1.00\end{array}$ \\
\hline & Universitarios & 4.27 & .98 & & Universitarios & 98 & $3.29 \quad 1.42$ \\
\hline \multirow{2}{*}{$\begin{array}{l}\text { Entrenadores } \\
\text { titulados }\end{array}$} & Secundarios & 4.39 & .96 & \multirow{2}{*}{$\begin{array}{c}\text { Instalaciones } \\
\text { limpias }\end{array}$} & & 13 & $\begin{array}{ll}4.85 & .38\end{array}$ \\
\hline & Universitarios & 4.06 & 1.10 & & Universitarios & 98 & $\begin{array}{ll}4.82 \quad .58 \\
\end{array}$ \\
\hline \multirow{2}{*}{$\begin{array}{l}\text { Entrenadores } \\
\text { amables }\end{array}$} & Secundarios & 4.85 & .38 & \multirow{2}{*}{$\begin{array}{c}\text { Instalaciones } \\
\text { disponibles }\end{array}$} & Secundarios & 13 & $\begin{array}{ll}4.54 & .78\end{array}$ \\
\hline & Universitarios & 4.68 & .71 & & Universitarios & 98 & $\begin{array}{l}4.46 \quad .74 \\
\end{array}$ \\
\hline \multirow{2}{*}{ Zona social } & Secundarios & 4.31 & .85 & & & & \\
\hline & Universitarios & 3.74 & 1.27 & & & & \\
\hline
\end{tabular}

\section{Criterios de motivación en función de la ocupación}

Respecto a los criterios de motivación en función de la ocupación de los encuestados (ver tabla 7), en primer lugar, en el caso de los empleados, el aspecto más valorado es que las instalaciones estén limpias $(4.81 \pm .58)$ y el menos valorado que tenga cafetería $(3.37 \pm 1.39)$. En el caso de los desempleados, el equipamiento (5.00 \pm .00$)$, que tenga entrenadores titulados $(5.00 \pm .00)$ y amables $(5.00 \pm .00)$ y que las instalaciones estén limpias $(5.00 \pm .00)$ y con buena disponibilidad (5.00土.00) son los aspectos más valorados, mientras que el hecho de que tenga cafetería $(3.33 \pm 2.08)$ y que organicen campeonatos $(3.33 \pm 2.08)$ son los menos valorados. Posteriormente, encontramos a los estudiantes, para quienes la limpieza de las instalaciones es el elemento más valo- 
rado $(4.77 \pm .60)$ mientras que el menos valorado es, de nuevo, que el centro tenga cafetería $(3.46 \pm 1.33)$. Finalmente, para los jubilados, el aspecto con una mayor media es que el centro tenga la última tecnología $(5.00 \pm .00)$, que los entrenadores sean amables $(5.00 \pm .00)$ y que las instalaciones estén limpias $(5.00 \pm .00)$, mientras que el aspecto con menor media es que el centro tenga cafetería ( $3.00 \pm 1.83)$. En cuanto a la comparación de grupos, no existen diferencias en ningún caso.

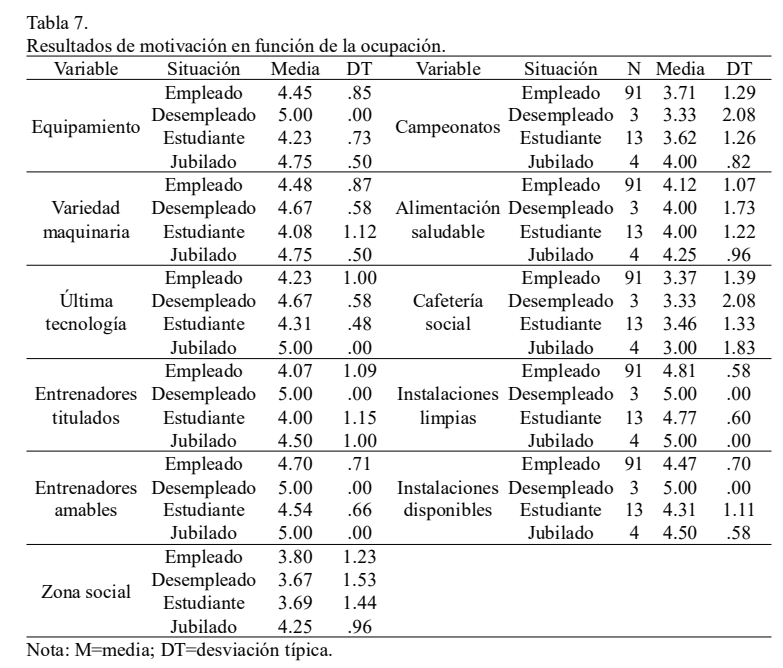

Criterios de motivación en función del nivel socioeconómico

La última variable sociodemográfica analizada, es el nivel socioeconómico que dicen tener los encuestados. En primer lugar, en la tabla 8 podemos ver que el aspecto más valorado por los que se consideran de nivel medio-bajo y de nivel medio es la limpieza de instalaciones, con valores de 4.89 ( \pm .32$)$ y $4.80( \pm .60)$, respectivamente, mientras que para los de nivel alto, los aspectos son, de nuevo, la limpieza (5.00

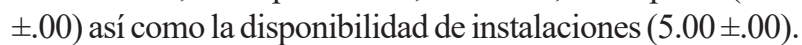
Por otro lado, respecto a los elementos menos valorados, los de clase media-baja y clase media eligen el servicio de cafetería con valores de $3.50( \pm 1.54)$ y de $3.38( \pm 1.34)$, respectivamente, mientras que para los de clase alta los elementos menos valorados son que el centro tenga zona social $(2.33 \pm 2.31)$, que organice campeonatos $(2.67 \pm 2.08)$ y que tenga cafetería $(2.33 \pm 2.31)$. En cuanto a la comparación de grupos, encontramos diferencias significativas en la variable de variedad

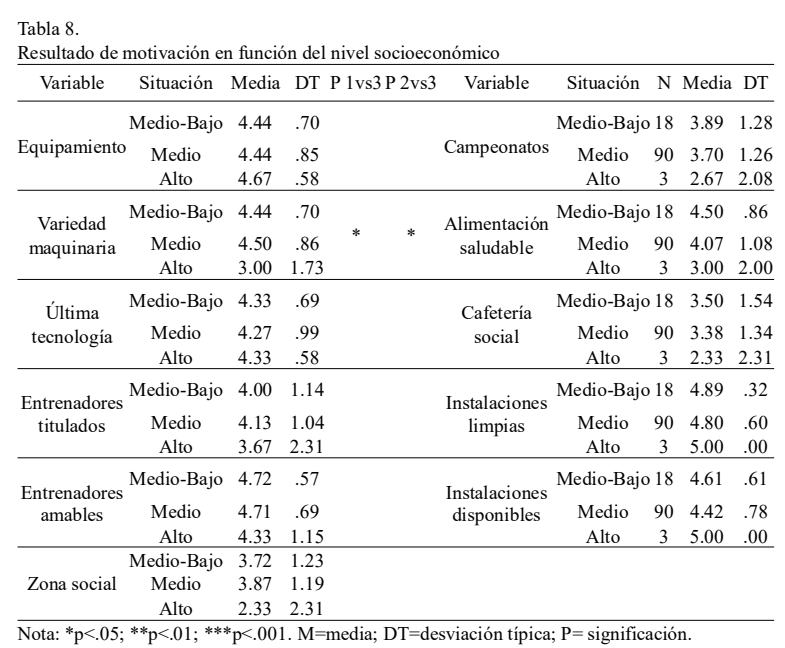

de maquinaria, entre los encuestados de nivel socioeconómico medio-baja y los de nivel alto $(p<.05)$ y entre los de nivel medio y los de nivel alto $(\mathrm{p}<.05)$ donde las puntuaciones de mayor a menor aparecen en los de nivel medio-bajo, nivel medio y nivel alto, respectivamente.

Una vez vistos los diferentes resultados, a continuación, en la tabla 9, se indica un resumen visual de los aspectos más destacables tanto en relación a los criterios de importancia como a los criterios de motivación, diferenciando en función de los aspectos sociodemográficos.

\begin{tabular}{|c|c|c|c|c|}
\hline \multirow{2}{*}{\multicolumn{3}{|c|}{ Criterios Importancia }} & \multirow{2}{*}{\multicolumn{2}{|c|}{$\begin{array}{l}\text { Criterios Motivación } \\
+\end{array}$}} \\
\hline & & & & \\
\hline Hombre & $\begin{array}{l}\text { Cerca domicilio y } \\
\text { multidisciplinar }\end{array}$ & $\begin{array}{l}\text { Pequeño y centro } \\
\text { comercial }\end{array}$ & Limpieza & Cafertería \\
\hline Mujer & $\begin{array}{c}\text { Cerca domicilio y } \\
\text { entrenador personal }\end{array}$ & $\begin{array}{l}\text { Público y centro } \\
\text { comercial }\end{array}$ & Limpieza & Cafetería \\
\hline $\begin{array}{c}\text { Estudios } \\
\text { Secundarios }\end{array}$ & Multidisciplinar & Pequeño & $\begin{array}{c}\text { Entrenadores amables y } \\
\text { Limpieza }\end{array}$ & Cafetería \\
\hline $\begin{array}{c}\text { Estudios } \\
\text { Universitarios } \\
\end{array}$ & Cerca domicilio & Pequeño & Limpieza & Cafetería \\
\hline Empleado & Cerca domicilio & Pequeño & Limpieza & Cafetería \\
\hline Desempleado & $\begin{array}{c}\text { Grande y } \\
\text { multidisciplinar }\end{array}$ & Público y pequeño & $\begin{array}{c}\text { Limpieza, equipamiento, } \\
\text { entrenadores amables y } \\
\text { titulados }\end{array}$ & $\begin{array}{c}\text { Cafetería y } \\
\text { campeonatos }\end{array}$ \\
\hline Estudiante & Cerca domicilio & Pequeño & Limpieza & Cafetería \\
\hline Jubilado & $\begin{array}{c}\text { Entrenador personal, } \\
\text { actividades dirigidas } \\
\text { y cerca domicilio }\end{array}$ & Cerca trabajo & $\begin{array}{c}\text { Limpieza, última } \\
\text { tecnología y entrenadores } \\
\text { amables }\end{array}$ & Cafetería \\
\hline $\begin{array}{l}\text { Económico } \\
\text { Medio-Bajo }\end{array}$ & Multidisciplinar & Centro comercial & Limpieza & Cafetería \\
\hline $\begin{array}{l}\text { Económico } \\
\text { Medio }\end{array}$ & Cerca domicilio & Centro comercial & Limpieza & Cafetería \\
\hline $\begin{array}{l}\text { Económico } \\
\text { Medio-Alto }\end{array}$ & $\begin{array}{l}\text { Grande y cerca } \\
\text { domicilio }\end{array}$ & $\begin{array}{l}\text { Alejado centro } \\
\text { ciudad }\end{array}$ & $\begin{array}{c}\text { Limpieza y } \\
\text { disponibilidad } \\
\text { instalaciones }\end{array}$ & $\begin{array}{c}\text { Zona social, } \\
\text { campeonatos y } \\
\text { cafetería }\end{array}$ \\
\hline
\end{tabular}

\section{Discusión}

La investigación realizada ha tenido como objetivo conocer la opinión de los usuarios de centros deportivos sobre qué aspectos consideran importantes y cuáles de ellos les motivan para inscribirse en uno de dichos centros deportivos, diferenciando las opiniones en función de las diferentes características sociodemográficas: género, nivel de estudios, ocupación y nivel socioeconómico. El estudio de las variables sociodemográficas como elementos a tener en cuenta para entender los fenómenos de comportamiento del consumidor, es algo ya habitual en la literatura científica, lógicamente también en el contexto deportivo, donde encontramos ejemplos que se han basado en ese tipo de análisis para analizar, por ejemplo, la imagen de marca en servicios deportivos (Alguacil, Crespo-Hervás \& Pérez-Campos, 2020) la segmentación en servicios fitness (Valcarce \& Díez, 2017) o las preferencias de los consumidores en relación al turismo deportivo (Slak-Valek, Shaw \& Bednarik, 2014). Este tipo de análisis permite la segmentación de los consumidores (Nuviala et al., 2014), conociendo los distintos perfiles de usuario y favoreciendo un mejor y más detallado entendimiento de los procesos. Dicha segmentación permite, además, que los centros puedan diferenciarse, ya sea mediante políticas de precios o campañas publicitarias adaptadas a cada target (Elasri, Triadó \& Aparicio, 2016). Parece lógico que conocer los aspectos que los usuarios consideran importantes puede ser útil a nivel de gestión, es por ello por lo que análisis como el análisis de importancia-valoración ha sido utilizado en la literatura para entender mejor los servicios (Ábalo, Varela \& Rial, 2006) así como los comportamientos de los consumidores (Lee \& Chae, 2008). Este tipo de estudios los encontramos también en el ámbito deportivo, 
permitiéndonos conocer que, aspectos como la variedad de actividades y los entrenadores personales son importantes para los usuarios, así como la higiene (León-Quismondo, García-Unanue \& Burillo, 2020). Estos datos concuerdan con los obtenidos en este estudio, ya que la higiene, además de que el centro fuese multidisciplinar, tuviese actividades guiadas y dispusiese de entrenadores personales que además fuesen amables, han sido aspectos resaltados por los usuarios.

Por otro lado, encontramos el análisis de las motivaciones para inscribirse a un centro deportivo. En este sentido, encontramos aportaciones como la de Quesada y GómezLópez (2017), en la que se lleva a cabo un análisis de los distintos perfiles motivacionales de los usuarios de centros deportivos, realizando un análisis de clústeres donde aparecen como motivaciones para la asistencia a centros deportivos aspectos como las ganas de divertirse y fomentar relaciones sociales, la salud o la apariencia. Otros estudios, como el de Arias-Ramos, Serrano-Gómez y García-García (2016), llevan a cabo un análisis diferenciando las opiniones de usuarios de un centro público y uno privado, obteniendo como conclusiones que los ítems que más destacan tienen que ver con el aspecto del personal, tanto en relación con la profesionalidad como con la amabilidad. Esto encaja con lo obtenido en nuestro estudio, donde el hecho de tener entrenadores amables era uno de los aspectos destacados.

En el presente estudio se puede identificar que, tanto para hombres y mujeres el principal factor que motiva a unirse a un centro deportivo es la limpieza, coincidiendo con el estudio de Alonso, Rial y Rial (2013) en el ámbito universitario, y el de Zamorano-Solís y García- Fernández (2018) con usuarios de centros de fitness. Por otra parte, se encuentran discrepancias con el estudio de Zamorano-Solís y GarcíaFernández (2018), ya que las mujeres no valoran como importante un trato personalizado, mientras que en el presente estudio uno de los criterios más importantes para acudir a un centro fue que éste tuviese entrenadores personales. Así mismo, se puede identificar que, en todos los segmentos analizados como en el nivel de estudios, ocupación, y nivel socioeconómico, el criterio que más motiva a los usuarios para dirigirse a un centro deportivo vuelve a ser la limpieza de las instalaciones. No obstante, en cuanto a los segmentos de nivel de estudios y nivel socioeconómico, los individuos que poseen estudios secundarios y los de nivel medio bajo, respectivamente, afirman que el criterio más importante para dirigirse a un centro deportivo es que sea multidisciplinar, coincidiendo con el estudio Arias-Ramos et al. (2016) en el que analizan las valoraciones de centros fitness públicos y privados. Estos mismos autores, afirman que, tanto en instalaciones públicas como privadas, los atributos más importantes se encuentran relacionados con el personal, lo que también se refleja en parte en la presente investigación.

El hecho de poder analizar estos aspectos comentados en función de los distintos perfiles de usuarios, no solo permite que podamos segmentar estrategias, sino que, consecuentemente, podremos mejorar aspectos que se sabe que son influyentes en el comportamiento de los consumidores. En este sentido, si conseguimos llegar a los usuarios atendiendo a aquello que les parece importante y les motiva, tendrán la sensación de que nuestro servicio encaja con lo que buscan, por lo que habrá actitudes más positivas hacia el servicio. Estas actitudes son buen indicador de las intenciones que van a tener los usuarios (Seo, Green, Ko, Lee \& Schenewark, 2007) y han mostrado tener un papel mediador a la hora de explicar su lealtad hacia el servicio (Sivarajah, 2014). Por tanto, ese conocimiento de la información sería un buen paso previo para desencadenar el resto de procesos.

\section{Conclusiones}

Este trabajo, que analiza las percepciones de los usuarios de centros deportivos, proporciona resultados útiles, tanto en relación a los aspectos importantes para ellos como para su motivación para elegir un centro deportivo. Tras los resultados podemos concluir, respecto al género, que no existen criterios diferentes en cuanto a la motivación de acudir al centro deportivo o no, sin embargo, en cuanto a la importancia de determinados aspectos, las mujeres valoran más que dispongan de entrenamientos personales y actividades dirigidas. Por tanto, los gestores que quieran orientarse a un mercado predominantemente femenino, podrán tener esos aspectos en cuenta. Por otro lado, respecto al nivel de estudios, se concluye que los que tienen estudios secundarios valoran más que sea un centro deportivo público y que no tenga grandes dimensiones, así como que disponga de una cafetería social y una alimentación saludable. En función de la ocupación, se concluye que son los habitantes que están jubilados los que valoran de forma más positiva, y en el momento de tener que elegir un centro deportivo, elegirían aquellos que son de tamaño reducido y donde sólo se pueda practicar una disciplina. En cambio, en cuanto a las motivaciones para acudir a estos servicios deportivos, no se han encontrado aspectos destacables en relación a la ocupación de los residentes. Por último, en cuanto al nivel socio-económico, tampoco existe ningún aspecto destacable que un grupo considere importante, sin embargo, sí que la variedad de maquinaria motiva a los usuarios con un nivel económico medio. Por lo tanto, este trabajo nos permite obtener información concreta de los distintos perfiles, lo que facilita la gestión de un centro deportivo y la toma de decisiones, favoreciendo la viabilidad de dicho servicio.

\section{Implicaciones, limitaciones y líneas futuras}

Estudios como el que aquí se presenta, suponen una contribución a la literatura sobre gestión deportiva, concretamente en lo relacionado con el perfil de los consumidores y sus criterios de importancia y motivación para inscribirse a un centro deportivo. Este tipo de análisis aporta a los gestores información útil acerca de cuáles pueden ser las preferencias de los distintos perfiles de usuarios, lo que permitirá ir comprendiendo en mayor medida el comportamiento del consumidor y, consecuentemente, poder segmentar de forma más eficaz, aumentando el éxito de las estrategias que se planteen. En cuanto a las limitaciones, encontramos en primer lugar la contextualización de los datos, ya que se centran en una ciudad y su área metropolitana, sin tener en cuenta otros contextos. Por otro lado, la consecución de una mayor muestra ayudaría para obtener una mayor representatividad, aunque la muestra actual ha permitido llevar a cabo los aná- 
lisis y conocer la temática en el contexto elegido, en el que prácticamente no hay datos al respecto. En cuanto a las futuras líneas de investigación, que solucionarían las limitaciones del presente estudio, se deberían llevar a cabo análisis similares más extensos, atendiendo en ese caso al tipo de centro, a su posicionamiento en precio o al país donde se encuentra, para poder observar si existen diferencias en función de esas distintas tipologías y contextos. Los resultados obtenidos, permitirían conocer una información más detallada y generalizable en este sentido.

\section{Referencias}

Ábalo, J., Valera, J., \& Rial, A. (2006). El análisis de importanciavaloración aplicado a la gestión de servicios. Psicothema, 18(4), 730-737.

Alguacil, M., Crespo-Hervás, J., \& Pérez-Campos, C. (2020). Sociodemographic analysis of brand perception in a public sports service: from target to person buyer. Retos-Nuevas Tendencias en Educación Física, Deporte y Recreación, 37, 139-146.

Alonso,D., Rial,J., \& Rial,A.(2013). Evaluación dela calidad percibida de los servicios deportivos en el ámbito universitario. Revista de Psicología del Deporte, 22(1), 143-150.

Arias-Ramos, M., Serrano-Gómez, V., \& García-García, O. (2016). ¿Existen diferencias en la calidad percibiday satisfacción del usuario que asiste a un centro deportivo de titularidad privada o pública? Un estudio piloto. Cuadernos de Psicología del Deporte, 16(2), 99-110.

Brady, M. K., Knight, G. A., Cronin Jr, J. J., Tomas, G., Hult, M., \& Keillor,B.D. (2005). Removing the contextual lens: Amultinational, multi-setting comparison of service evaluation models. Journal of Retailing, 81(3), 215-230.

Campos, F., Martins, F., Simões, V., \& Franco, S. (2017). Fitness participants perceived quality by age 435 and practiced activity. Journal of PhysicalEducation and Sport,17(2),436698.doi:10.7752/ jpes.2017.02105.

Desbordes, M., Ohl,F., \& Tribou, G. (2001). Estrategias del marketing deportivo. Análisis del consumo deportivo. Barcelona: Paidotribo.

Elasri, A., Triadó, X. M., \& Aparicio, M. (2016). Los usuarios de los centros deportivos públicos: una aproximación de segmentación sobre hábitos deportivos y satisfacción. Revista de Psicología del Deporte, 25(3), 15-18.

Frederick, C.M., \& Ryan, R.M.(1993). Differences in motivation for sport and exercise and their relations with participation and mental health. Journal of Sport Behavior, 16, 124-146.

García-Fernández, J., Fernández-Gavira, J., \& Bernal, A. (2014). La percepción de calidad y fidelidad en clientes de centros de fitness low-cost. Suma Psicológica, 21(2), 123-130.

García-Fernández, J., Gálvez, P., Bernal, A., \& Vélez, L. (2016). El gasto económico en centros de fitness low-cost: Diferencias según fidelidad y características del cliente. Sport TK: Revista Euroamericana de Ciencias del Deporte, 5, 137-144.

Lee, S. J., \& Chae, I. S. (2008). Expectation and Satisfaction of Foreign Customers Visiting Korean Restaurants Located in USA; Analysis for Food and Service Attributes through IPAAnalysis. Journal of the Korean Society of Food Culture, 23(2), 152-162.

León-Quismondo, J., García-Unanue, J., \& Burillo, P. (2020). Service Perceptions in Fitness Centers: IPAApproach by Gender and Age. International Journal of Environmental Research and Public Health, 17(8), 1-10.

Luna-Arocas, R. (1998). Segmentación psicográfica y marketing deportivo. Revista de Psicología del Deporte, 13, 121-133.

Luna-Arocas, R. Mundina, J., \& Quintanilla, I. (1997). Marketing so- cial aplicado al deporte: una nueva conceptualización. En S. Camarero, V. Tella, \& J.J. Mundina (comp.) Análisis de la práctica deportiva. Una visión multidisciplinar. Valencia: Promolibro.

Luna-Arocas, R., \& Li-Ping Tang, T. (2005). The use of cluster analysis to segment clients of a sport center in Spain. European Sport Management Quarterly, 5(4),381-413.

Martínez, J.R., \& Balbastre, F. (2004). La gestión en los Servicios Deportivos Municipales: de la calidad en el servicio a la calidad en la gestión. El caso de las piscinas cubiertas en la FDM de Valencia. Investigacióny Marketing, 83, 22-30.

Molina, N., Mundina, J., \& Gómez, A. (2019). Perfil del usuario de centros deportivos privados, según género, edad y nivel de antigüedad. SPORTTK-Revista EuroAmericana de Ciencias del Deporte, 8(1), 23-28.

Mundina, J., \& Calabuig, F. (1999). El Marketing Social al Serviciodela Gestión de Calidad: El Caso de los Servicios Públicos Náuticos de la Generalitat Valenciana. Apunts, 57, 77-83.

Nuviala, R. N., Teva-Villén, M. R., Pérez-Ordás, R., Grao-Cruces, A., Fajardo, J.A. T., \& Nuviala,A. N. (2014). Segmentación de usuarios de servicios deportivos. Retos. Nuevas tendencias en Educación Física, Deporte y Recreación, (25),90-94.

Quesada, D., \& Gómez-López, M. (2017). Perfiles motivacionales de los usuarios de un centro deportivo público. Journal of Sport \& Health Research, 9(1), 85-96.

Rial,A., Lamas, L., \& Varela, J. (2009). Aplicación de los modelos de ecuaciones estructurales a la identificación de las variables predictoras de la fidelidad a un centro comercial. Anales de Psicología, 25(1), 134-141.

Rial, J., Varela, J., Rial,A., \& Real, E. (2010). Modelizacióny medida de la Calidad Percibida en centros deportivos: la escala QSport-10. Revista Internacional de Ciencias del Deporte, 18(6), 57-73

Ros-Castelló,P., Calabuig, F., \& Tafalla,A. (2019). Perfil del usuario de la fundación deportiva municipal de valencia y sus hábitos de práctica deportiva. SPORTTK-Revista EuroAmericana de Ciencias del Deporte, $8(1), 17-22$.

Ryan, R., Frederik, C., Lepes, D., Rubio, N., \& Kennon, S. (1997). Intrinsic motivation and exercise adherence. International Journal of Sport Psychology, 28, 335-354.

Seo, W. J., Green, B. C., Ko, Y. J., Lee, S., \& Schenewark, J. (2007). The effect of web cohesion, web commitment, and attitude toward the website on intentions to use NFL teams' websites. Sport management review, 10(3), 231-252.

Sicilia, Á., González-Cutre, D., Artés, E. M., Orta,A., Casimiro,A. J., \& Ferriz, R. (2014). Motivos de los ciudadanos para realizar ejercicio fisico: un estudiodesde la teoría dela autodeterminación. Revista Latinoamericana de Psicología, 46(2), 83-91.

Sivarajah, R. (2014). The impact of consumer experience on brand loyalty: the mediating role of brand attitude. International Journal of Management and Social Sciences Research, 3(1), 73-79.

Slak-Valek, N., Shaw, M., \& Bednarik, J. (2014). Socio-demographic characteristics affecting sport tourism choices: Astructural model. Acta gymnica, 44(1), 57-65.

Thogersen, C., Lane, H. J., Biscomb, K., Jarrett, H., \& Lane, A. M. (2007). Women's motives to exercise. Women in Sport \& Physical Activity Journal, 16(1), 16-27.

Triadó, X.M., \&Aparicio, P. (2004). El tipo de cuota como variable de fidelización de usuarios en entidades deportivas. Investigación y Marketing, 83,31-38.

Valcarce, M., \& Díez-Rico, C. (2017). Segmentación, redes sociales y edad en el sector del fitness. Journal of Sports Economics \& Management, 7(2), 111-119.

Zamorano-Solís, S., \& García-Fernández, J. (2018). El análisis importancia-valoración según género y permanencia: el caso de los centros de fitness. Materiales para la Historia del Deporte, (16), 2435 . 